Revue française de la traduction

\title{
L'art de la traduction de l'humour dans la littérature pour enfants : la traduction du Petit Nicolas en persan
}

Sevil Zeynaligargari et Farideh Alavi

\section{(2) OpenEdition \\ Journals}

Édition électronique

URL : http://journals.openedition.org/traduire/243

DOI : $10.4000 /$ traduire. 243

ISSN : 2272-9992

Éditeur

Société française des traducteurs

Édition imprimée

Date de publication : 1 juin 2011

Pagination : 90-108

ISSN : 0395-773X

\section{Référence électronique}

Sevil Zeynaligargari et Farideh Alavi, «L'art de la traduction de l'humour dans la littérature pour enfants : la traduction du Petit Nicolas en persan », Traduire [En ligne], 224 | 2011, mis en ligne le 03 février 2014, consulté le 30 avril 2019. URL : http://journals.openedition.org/traduire/243 ; DOI : $10.4000 /$ traduire.243 


\title{
L'art de la traduction de l'humour dans la littérature pour enfants : la traduction du Petit Nicolas en persan
}

\author{
Sevil Zeynaligargari, Farideh Alavi
}

La traduction des textes humoristiques constitue depuis toujours l'une des inquiétudes des traducteurs et l'" intraduisibilité " ou la " traduisibilité " de ces genres littéraires a toujours fait l'objet de débats. Cette inquiétude semble plus grave quand il s'agit de la traduction de textes humoristiques destinés à des lecteurs aussi particuliers que les enfants. Le Petit Nicolas est une série de récits illustrés et humoristiques créée par deux humoristes de génie : René Goscinny, qui écrit les textes, et Jean-Jacques Sempé, qui réalise les dessins. Cette œuvre traduite en persan a été bien accueillie par les enfants iraniens. Le présent article se propose d'étudier les stratégies appliquées par les traductrices du Petit Nicolas pour rendre le côté humoristique du texte original. Pour ce faire, nous nous appuierons sur les théories de la traduction, et particulièrement la théorie de Nida relative à l'u équivalence dynamique ".

La littérature pour enfants se constitue au croisement du livre d'image et de l'éducation. Cette littérature apparaît en Europe dans la seconde moitié du xvIIIe siècle et implose au xxe siècle. Ce qui est important dans la littérature pour enfants, c'est son " lecteur ". C'est la particularité qui sépare cette littérature de celle des adultes. Et cette différence est plus évidente quand il s'agit d'humour, à cause des frontières qui existent entre l'humour à l'adresse des enfants et l'humour à l'adresse des adultes.

L'humour est un sujet très délicat dans la littérature pour enfants et présente des types très particuliers. Le fait d'écrire des textes humoristiques pour enfants est une tâche difficile qui implique une vaste connaissance du monde des enfants, de leur psychologie et de leur langage. En Iran, il y a peu d'écrivains spécialistes de ce domaine. Il y a un long chemin à parcourir pour connaître la vraie place de ce genre d'écriture dans la littérature pour enfants. Ce qui est important dans la traduction des textes humoristiques en général, c'est le transfert du côté humoristique, qui exige une connaissance approfondie de la langue et de la culture cible. D'ailleurs, les traducteurs des littératures pour enfants sont souvent confrontés à des difficultés comme l'identification du lecteur, l'adaptation du texte avec la situation du pays d'accueil ou 
encore le choix des termes et du vocabulaire selon la connaissance linguistique des enfants. Un texte très réussi dans ce domaine est la série du Petit Nicolas, réalisée par Sempé et Goscinny.

Le Petit Nicolas contient 19 courts récits, chacun constitué de 8 à 9 pages. Dès la première page, le lecteur entre dans l'histoire. Les récits sont racontés par un garçon du nom de Nicolas (âgé de 8 à 10 ans), dans un langage humoristique. II décrit ses aventures avec ses copains, à l'école ou dans sa famille, avec un langage à la fois imagé et enfantin. Ce langage imagé est l'une des raisons pour lesquelles cette œuvre a été adaptée au cinéma.

La première histoire du Petit Nicolas paraît dans Sud-Ouest Dimanche en 1959. La même année, Le Petit Nicolas paraît dans Pilote, dès le premier numéro, aux côtés d'Astérix. En 1960, Le Petit Nicolas paraît sous forme de livre aux éditions Denoël.

Dans cet article, nous examinerons les dimensions humoristiques de deux traductions en persan du Petit Nicolas, réalisées par Vida Saâdat, septième édition en 2001 (ci-après désignée par l'abréviation T1) et Âfâgh Hâmed Hâshemi, deuxième édition en 2005 (ci-après désignée par l'abréviation T2), toutes deux basées sur la publication de 1960 du Petit Nicolas. Ces deux traductions sont plus accessibles que les autres.

L'humour est souvent considéré comme intraduisible ou difficile à traduire. La traduction de l'humour peut sembler compromise parce que ce qui paraît drôle dans une culture pourrait sembler sérieux dans une autre. II s'agira pour nous de compléter la théorie de Nida sur l'équivalence dynamique afin d'avancer certains éléments théoriques à propos de la traduction des textes humoristiques pour enfants. Ainsi, nous avons tenté, tout au long de ce travail, de présenter un début de théorisation de la traduction des textes humoristiques pour enfants.

\section{Les jeunes lecteurs et l'humour}

L'humour est une critique des problèmes sociaux et individuels, mais qui a pour but d'améliorer les situations. II est évident que les critiques sont parfois amères. L'humour est un bon moyen de révéler les laideurs de la vie et les situations désagréables.

Robert Escarpit propose la définition suivante de l'humour :

[L'humour] est une volonté et en même temps un moyen de briser le cercle des automatismes que, mortellement maternelle, la vie en société et la vie tout court cristallisent autour de nous comme une protection et comme un linceul. (Escarpit, 1994, p. 127).

Selon la définition d'Escarpit, l'humour essaie de peindre la frontière entre la vie parfaite et la vie réelle et à montrer la différence avec ce qui est la réalité cachée de la vie. L'humour est en effet l'une des formes du comique. Henri Bergson, philosophe français, décrit dans son ouvrage 


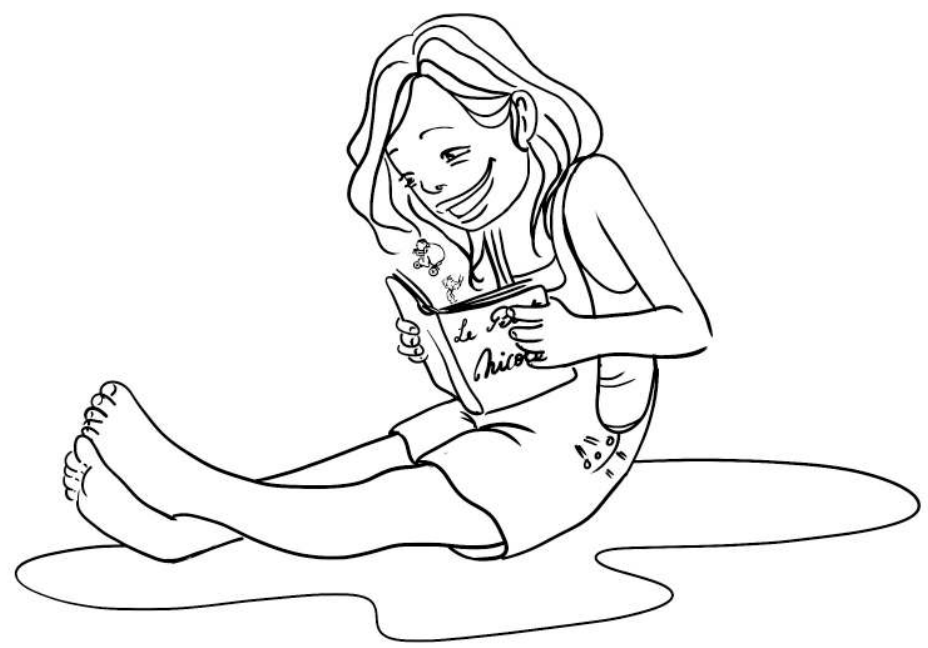

Illustration : Marlène Junius, http://alotoftralala.over-blog.com

Le rire, essai sur la signification du comique (Bergson, 1959, p. 9-33) différentes approches théoriques du rire et du comique à partir desquelles nous tirons les conclusions ci-dessous.

1. Le comique ne se produit que pour ce qui est dit proprement humain. La réussite des comédiens découle de ce simple principe.

2. L'émotion est le plus grand ennemi du rire. Pour être pleinement sensible à l'humour, le téléspectateur ou le lecteur devra être dans un état d'indifférence avant de lire ou voir un sujet comique.

3. Le comique s'adresse à l'intelligence, donc il a besoin d'une intelligence, d'un trait d'esprit.

4. La distraction et les préoccupations ne permettent pas de rire.

5. Toute difformité peut provoquer le rire (comme dans les caricatures).

6. Tous les événements qui attirent l'attention sur l'apparence, mais font évoluer la pensée, peuvent être drôles.

7. Tout ce qui ressemble au " jeu du diable à ressort " engendre une situation comique en présentant des actions bizarres.

8. L'une des dimensions inévitables du comique est le langage et ce langage doit être plein d'ironie, de satire et de plaisanterie. 
D'après la théorie de Bergson, le comique a besoin d'une intelligence. II change selon le niveau de cette intelligence. Par exemple, l'humour politique ne peut pas être utilisé dans les ouvrages pour enfants. La langue est aussi l'un des outils de l'humour. La langue humoristique s'adresse à l'intelligence et change selon le groupe d'âge du lecteur.

Tout le monde aime faire rire les enfants, mais il faut le faire d'une manière correcte et professionnelle. Les textes humoristiques sont l'un de ces procédés. Les enfants lisent les mots et rient après avoir découvert ce qui est caché derrière ces mots. Les enfants apprécient l'humour dans la littérature qui leur est destinée. "II permet d'apprivoiser les peurs, de dédramatiser les choses graves, de faire passer un message difficile, de transgresser les règles établies. "(1). II faut que les humoristes s'approchent du monde des enfants et se familiarisent avec leurs inquiétudes. Ils doivent connaître le langage des enfants de leur époque.

L'humoriste pour enfants choisit les sujets qui leur sont les plus familiers et les plus proches, notamment les jeux, l'école, les copains, ou parfois les animaux que les enfants connaissent bien. Le monde des animaux est plus attirant pour les enfants. On peut utiliser cette particularité pour leur présenter des textes humoristiques. Par exemple, les animaux qui ont un physique et un aspect particuliers comme la girafe avec son long cou ou la grenouille avec ses yeux gonflés et sa grande bouche sont des sujets de "rigolade " pour les enfants. Le langage de l'humour à l'adresse des enfants doit leur être compréhensible et doit donc être adapté à leurs connaissances. Le savoir linguistique des enfants est limité : la langue utilisée doit être claire et exempte d'argot, alors que dans l'humour à l'adresse des adultes, la langue a plus de liberté. Les sujets humoristiques pour enfants sont aussi limités. L'enfant ne connaît pas encore bien le monde réel. II est préférable que les textes pour enfants, qu'il s'agisse de poèmes ou de récits, soient en harmonie avec leur monde et les familiarisent avec la simplicité et la joie de la vie. La série de récits du Petit Nicolas est un ouvrage humoristique très réussi de la littérature pour enfants, qui a respecté à peu près toutes les particularités de l'humour. Ce livre a dépassé les frontières de la France et s'est fait connaître dans les autres pays. Nous allons analyser sa construction textuelle afin de découvrir l'effet de cet aspect sur le côté humoristique du livre.

\section{La construction textuelle (syntaxique) du Petit Nicolas}

\section{Les phrases simples}

"La phrase simple est constituée d'une seule proposition et vise souvent à rendre compte de la réalité immédiate, non altérée ou mise en regard du récitant. " (Fromilhague et Sancier, 1991, p. 180). Dans Le Petit Nicolas, l'appréhension naïve et spontanée du monde est bien rendue.

(1) http://www.crdp.ac-creteil.fr/telemaque/comite/JPGourevitch.htm 
Les phrases courtes et simples utilisées représentent un univers simple, voire nailf. On n'y trouve aucune structure complexe :

Aujourd'hui, à l'école, la maîtresse a manqué. Nous étions dans la cour, en rangs, pour entrer en classe, quand le surveillant nous a dit : "Votre maitresse est malade, aujourd'hui. " (p. 22)

L'une des particularités des phrases destinées aux enfants est leur brièveté. Les enfants ne peuvent pas saisir le sens et le message des phrases longues. Elles les ennuient et leur font perdre le plaisir de la lecture. Voici comment Nâder Ebrâhimi, dans Écrire en persan pour les enfants (Ebrâhimi, 1974, p. 30-33), résume les particularités des phrases destinées aux enfants :

Il faut éviter les petites phrases dans une phrase ainsi que les petites phrases qui viennent l'une après l'autre sans pause, les jeux de mots, les lexiques qui se suivent sans pause et de nombreux changements dans la structure des phrases.

Sachant que Le Petit Nicolas est un récit humoristique qui nécessite un rythme rapide et joyeux, il faut à tout prix conserver cette particularité dans les traductions persanes.

\section{La parataxe asyndétique}

L'utilisation des éléments de coordination donne une dynamique et une vivacité à l'énoncé. Les pronoms relatifs "qui " et " que ", la conjonction de coordination " et " sont largement utilisés dans Le Petit Nicolas. En voici un exemple:

Alors, Clotaire s'est levé et il a dit qu'il allait faire manger son livre d'arithmétique à Agnan, ce qui était vraiment une drôle d'idée, mais ça n'a pas plu à Agnan qui a crié : "Non! J'ai des lunettes! - Tu vas les manger aussi ! " a dit Clotaire, qui voulait absolument qu'Agnan mange quelque chose. (Sempé-Goscinny, 1960, p. 26)

Ces coordinations donnent un effet de rapidité au texte original, qu'il faut rendre dans la traduction. Mais il est parfois difficile, voire impossible, de transférer cet effet dans la langue cible à cause des différences entre les structures des deux langues.

\section{Le rythme}

Le rythme des récits du Petit Nicolas est rapide et donne une dynamique et une vivacité au texte. Cela produit une forme de ravissement chez le lecteur. On ne trouve pas de récits linéaires dans ce livre. Presque toutes les phrases contiennent une protase, une acmé et une apodose. L'acmé désigne le point le plus élevé de la phrase, entre la protase (montée de la phrase) et l'apodose (descente de la phrase). Ce changement du rythme des phrases attire l'attention des enfants et leur évite de s'ennuyer. Voici un exemple (le signe // désigne l'acmé) : 
Maman a regardé la fleur, elle avait l'air un peu étonnée, et puis, // elle m'a pris dans ses bras, // elle m'a embrassée des tas et des tas de fois, // elle a dit qu'elle n'avait jamais reçu un aussi beau bouquet et // elle a mis la fleur dans le grand vase bleu du salon. (Ibid., p. 72)

Dans cette phrase on constate un changement de rythme à quatre reprises. Ce changement attire l'attention des enfants et rend le récit plus intéressant. Le dialogue entre les personnages est aussi utilisé pour rythmer le récit.

On constate que le respect des éléments caractéristiques de la littérature enfantine attire les jeunes lecteurs de Goscinny. Le choix pertinent du narrateur, le rythme des récits, la brièveté, les répétitions et tous les autres éléments déjà cités produisent ensemble une œuvre humoristique agréable pour un jeune lecteur. Ces éléments aident à raconter le monde des enfants.

\section{Les types d'humour applicables dans les livres pour enfants}

Quatre formes d'humour (Mohébi, $n^{\circ} 552$ ) sont applicables dans les livres pour enfants:

\section{L'humour physique}

L'humour physique est utilisé pour les enfants de 5 à 7 ans. II s'agit de simples plaisanteries physiques et corporelles. Les enfants de ce groupe d'âge rient même à la plus petite plaisanterie. Cette sorte d'humour se retrouve particulièrement dans les bandes dessinées. Par exemple, dans Le Petit Nicolas, quand les copains commencent à se battre. Voici un exemple de ce type :

(... ) et Agnan a enlevé ses lunettes pour les essuyer, alors, Alceste en a vite profité et il lui a donné une gifle. (Sempé-Goscinny, 1960, p. 108)

Le fait de profiter de la situation et de donner une gifle fait rire les enfants. Le Petit Nicolas est plein de ces actions comiques entre copains, mais l'humour ne réside pas dans les querelles proprement dites. Dans l'exemple suivant :

Monsieur Bordenave avait l'air vraiment fâché et il est venu en courant vers nous, mais il n'est pas arrivé, parce qu'il a glissé sur le sandwich à la confiture d'Alceste, (...) (Ibid., p. 148)

La glissade de monsieur Bordenave nous fait rire. La glissade est une action comique, particulièrement quand la personne qui glisse est vraiment fâchée. Goscinny a bien profité de cette action physique pour déclencher le rire chez son lecteur.

\section{L'humour de situation}

Ce genre apparaît dans l'écriture. Une situation irréaliste et fantaisiste rend le récit humoristique. La parole est au service de la narration et de la description de situations drôles. Dans cet exemple, le père de Nicolas et Monsieur Blédurt se disputent pour monter sur le vélo de Nicolas : 
Papa et monsieur Blédurt ont tiré au sort et c'est monsieur Blédurt qui est parti le premier. Comme c'est vrai qu'il est assez gros, on ne voyait presque pas le vélo et les gens qui passaient dans la rue se retournaient en rigolant pour le regarder, monsieur Blédurt. (Ibid., p. 116)

Ce qui nous fait rire, c'est d'imaginer le gros homme assis sur un vélo d'enfant. Les dessins de Sempé aident les enfants à imaginer cette situation.

\section{L'humour caché dans les discours}

On l'utilise pour les groupes d'âge qui comprennent bien le sens caché de l'humour derrière les mots. Dans la littérature pour enfants, ce genre apparaît principalement sous forme de prose rimée et parfois sous forme de poésie. La structure des paroles engendre la situation humoristique. Comme dans cet exemple :

"Si tu ne veux pas rester en retenue, Agnan, a crié la maîtresse, je te prierai de garder tes réflexions pour toi ! " Agnan s'est mis à pleurer. "Vilain cafard " a crié quelqu'un, mais la maitresse n'a pas su qui c'était, sinon, j'aurais été puni, alors, (...) (Ibid., p. 63)

On n'attend pas cette phrase : " ..., sinon, j'aurais été puni... ". C'est comme le " diable à ressort " selon Bergson. Cette phrase inattendue nous fait rire.

\section{Les plaisanteries des personnages du récit}

Les personnages comiques de ces livres font ce que les enfants n'ont pas la permission de faire, ce qui attire l'attention des jeunes lecteurs. Dans les récits, l'un des éléments très importants qui doit évoluer selon l'âge des enfants, c'est la situation dans laquelle on met le personnage principal. Ce type d'humour est le plus difficile à manier dans les textes pour enfants. On peut citer cet extrait du Petit Nicolas, quand Alceste invite Nicolas à fumer un cigare :

Alceste, comme le cigare était à lui, c'était lui qui commençait, aspirait en faisant des tas de bruit et il y avait beaucoup de fumée. Le premier coup, ça l'a surpris, Alceste, ça l'a fait tousser et il m'a passé le cigare. J'ai aspiré, moi aussi, et, je dois dire que je n'ai pas trouvé ça tellement bon et ça m’a fait tousser, aussi. "Tu ne sais pas, m’a dit Alceste, regarde ! La fumée par le nez ! " Et Alceste a pris le cigare et il a essayé de faire passer la fumée par son nez, et ça, ça l'a rudement fait tousser. Moi, j'ai essayé à mon tour et j'ai mieux réussi, mais la fumée m'a fait piquer les yeux. On rigolait bien. (Ibid., p. 101)

L'écrivain laisse ses personnages appréhender le monde comme ils l'entendent. II n'intervient pas directement, il présente le résultat de l'action par la bouche de Nicolas:

C'est difficile de fumer le cigare quand on est petit ! (Ibid., p. 99)

Les deux premiers types sont moins efficaces que les deux derniers. Si toutes ces formes d'humour peuvent être utilisées dans les livres pour enfants destinés à tous les groupes d'âge, on note que les ouvrages qui ont le plus de succès sont ceux qui les emploient toutes à la fois. 


\section{Recréation ou traduction des textes humoristiques pour enfants}

Les différences culturelles et linguistiques entre deux langues ne permettent pas une traduction littérale ou tout à fait libre. Si on essaye de trouver un mot équivalent dans la langue d'arrivée (quand on rencontre un mot intraduisible dans le système culturel ou linguistique de la langue cible), la traduction devient impossible. Donc, c'est au niveau de la traduction lexicale qu'on rencontre le problème de l'intraduisibilité. Pour que le texte traduit soit compréhensible et acceptable pour le lecteur de la langue d'arrivée, on est obligé de garder une certaine liberté à l'égard du texte original.

La recréation est un autre aspect de la fidélité. Ce n'est pourtant pas un moyen de se débarrasser des problèmes de traduction. " La fidélité néglige souvent ce qui est derrière la traduction et considère ainsi la traduction comme une fin. Mais la recréation nous rappelle la fonction, le but et la tendance. " (Xiaoyi, 1999, p. 68). La notion de recréation peut bien justifier la traduisibilité par la recréation dans la traduction littérale.

Quand on traduit, on imagine un lecteur. Par conséquent, une traduction est toujours faite avec un objectif et pour un lecteur particulier. Le traducteur pour enfants est obligé de tenir compte des goûts et des capacités de ses lecteurs. Si l'on considère la traduction comme une communication, alors la traduction pour enfants équivaut à établir une communication entre les adultes et les enfants. Le destinataire de la littérature pour enfants, " plus qu'un lecteur réel, est une construction, un amalgame dans lequel se juxtaposent tous les narrataires que l'auteur peut avoir en tête au moment de l'écriture, ses propres enfants, l'enfant qu'il était (ou pense se souvenir qu'il était), l'enfant-lecteur idéal tel qu'il l'imagine, mais aussi les adultes prescripteurs ou accompagnateurs de la lecture du jeune. " (Douglas, 2007, p. 124).

Ritta Oittinen, professeur de traduction à l'université de Tampere (Finlande), déclare dans son ouvrage intitulé Translating for children que la traduction pour enfants doit être plus que la traduction des mots. Elle insiste sur l'aspect extralinguistique. Par exemple, les traducteurs de bandes dessinées traduisent toutes les situations qui constituent les mots, les images et la lecture à voix haute. Quand le traducteur saisit le sens de tous les mouvements, mots etc., il doit les interpréter. Selon Ritta Oittinen, le succès du traducteur réside dans cette interprétation.

Avant de commencer son travail, le traducteur doit clarifier l'objectif de sa traduction d'une œuvre particulière. Dans les textes humoristiques, la fidélité au style de l'auteur est-elle plus importante que la production du même effet sur le lecteur cible ? Posons alors cette question d'une autre manière : dans la traduction de l'humour, est-ce le lecteur ou le texte qui prime? La réponse à cette question nous conduit à rechercher une solution pour la traduction des textes humoristiques. Nous essayons de trouver notre réponse dans les théories de Nida, notamment dans sa définition de l'équivalence dynamique : 
Dynamic is therefore to be defined in terms of the degree to which the receptors of the message in the receptor language respond to it in substantially the same manner as the receptors in the source language. This response can never be identical, for the cultural and historical settings are too different, but there should be a high degree of equivalence response, or the translation will have failed to accomplish its purpose(2). (Nida, 1969, p. 24)

Selon cette définition, une bonne traduction est celle qui produit le même effet dans la langue d'arrivée que le texte original, c'est le sens de "l'équivalence dynamique " (Horri, 2006, p. 6). L'équivalence dynamique est " une équivalence qui ne se limite pas au plan formel, mais touche aussi les plans sémantique et pragmatique " (Jun, 1999, p. 17). Quand un traducteur essaye de trouver l'équivalence d'un texte, il ne le traduit pas littéralement. II exprime le sens en fonction des contraintes de la langue d'arrivée et de ses préférences stylistiques. Dans ce cas, on dit que le texte et sa traduction sont équivalents parce qu'on ne traduit pas le mot mais le sens. Avec cette forme de traduction, le transfert du message est très important. Le traducteur ne supprime pas le message, il le modifie pour mieux le rendre.

Si Abolfazlé Horri (Horri, 2006, p. 12) accepte que dans la traduction des textes humoristiques, le lecteur soit plus important que le texte, on pourra dire que le fait de trouver une équivalence n'est pas une sorte de fidélité à la structure du texte mais une sorte de fidélité aux effets produits par le texte original. Selon Nida, la traduction correspond à la définition suivante :

Translating [which] consists in producing in the receptor language the closest natural equivalent to the message of the source language, first in meaning, and secondly in style(3). (Nida, 1969, p. 12)

La plupart des traducteurs sont d'avis qu'il n'y a pas d'" équivalence exacte " (Solhdjo, 1998, p. 82) pour un mot; on utilise "l'équivalence la plus proche" (Ibid., p. 82). Par conséquent, le lecteur de la traduction saisit d'abord l'aspect humoristique du texte. Ensuite, la traduction doit produire le même effet sur lui que le texte original sur son lecteur.

Dans ce cas, on dit que le texte et sa traduction sont équivalents parce qu'on ne traduit pas le mot mais le sens. Dans cette forme de traduction, le transfert du message est très important ; le traducteur ne le supprime pas, il le modifie pour mieux le rendre. Voici un exemple :

Mais Alceste m'a dit que j'étais tombé sur la tête et que jamais un monsieur ne voudrait nous donner du feu parce qu'on était trop petit. " (Sempé-Goscinny, 1960, p. 98)

(2) La dynamique se définit donc par le degré de réponse au message du récepteur dans la langue de réception, cette réponse devant être plus ou moins équivalente à celle du récepteur dans la langue source. Les réponses ne peuvent jamais être identiques car les environnements culturels et historiques sont trop différents mais il doit y avoir un haut degré d'équivalence, ou la traduction aura manqué son but.

(3) L'acte traductionnel [qui] consiste à produire, dans la langue de réception, l'équivalent naturel le plus proche du message de la langue source, premièrement par le sens, et deuxièmement par le style. 
"ولى آلسست كفت: " مكر ديوانه اي؟ هيج وقت يك آقا حاضر نمى شود به ما آتش بدهد، براى /ينكه ما بجه

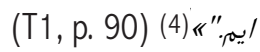

(5) (الما آلسست كفت كه منخ من عيب دارد و هيجج وقت يك آقا به ما كبريت نمى دهد خون ما خيلى بجه

هستيم." (T2, p. 105)

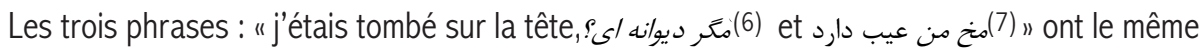
sens, mais les mots français et persans ne correspondent pas. Les mots n'ont pas tous été traduits en tant que tels mais le sens a été exprimé. Dans ce cas, on dit que cette phrase et ses traductions sont équivalentes.

Mais il y a deux raisons pour lesquelles le texte humoristique traduit ne produit pas le même effet sur le lecteur de la langue d'arrivée : les raisons intertextuelles et les raisons hypertextuelles, comme l'humour, qui a un arrière-plan culturel spécifique.

\section{L'humour basé sur la culture}

Nida (2001, p. 111) distingue trois approches théoriques de la traduction : philologique, linguistique et sémiotique. II classifie son approche parmi les approches linguistiques et insiste sur sa dimension culturelle. Selon Nida cité par Mounin :

Nida classe les problèmes posés par la recherche des équivalences - lors du passage d'un monde culturel à un autre au cours d'une traduction - selon cinq domaines : l'écologie, la culture matérielle (toutes les technologies au sens large, toutes les prises de l'homme sur le monde au moyen d'outil, d'action matérielle), la culture sociale, la culture religieuse et la culture linguistique. (Mounin, 1963, p. 62)

On peut dire que l'humour de chaque pays a une relation étroite avec son mode de vie (la culture matérielle) et sa région. C'est pour cette raison que chaque pays a son propre humour. Selon la culture, l'histoire, la société, la langue, la religion etc., les gens rient de sujets différents. La problématique de la culture dans les textes présente un défi particulier lors de la traduction. Le traducteur de textes humoristiques saisit tout d'abord le type d'humour utilisé dans le texte original. II doit le comparer avec la culture de la langue d'arrivée parce que le destinataire du message traduit est certainement quelqu'un dont la langue et la culture diffèrent de celles du destinataire original. II doit vérifier si ce genre d'humour a la même valeur dans la langue cible, ou s'il est plaisant pour le lecteur cible.

(4) " Mais Alceste a dit : "Tu es fou ? Jamais un monsieur ne voudra nous donner du feu parce qu'on est trop petit." "

(5) " Mais Alceste a dit que j'avais un problème dans le cerveau et que jamais un monsieur ne nous donnerait du feu parce qu'on était trop petit. "

(6) Tu es fou?

(7) J'ai un problème dans le cerveau. 
Voici un exemple d'humour culturel et de ses traductions dans Le Petit Nicolas:

... Clotaire a dit : "Regardez Alceste, maintenant c'est lui qui a l'air d'une andouille, avec les fleurs!" Alors, Alceste lui a donné un grand coup sur la tête, avec le bouquet. "Mes fleurs! J'ai crié. Vous allez casser mes fleurs ! " C'est vrai, aussi ! Alceste, il donnait des tas de coups avec mon bouquet et les fleurs volaient de tous les côtés parce que le papier s'était déchiré et Clotaire criait : "ça ne me fait pas mal, ça ne me fait pas mal! " Quand Alceste s'est arrêté, Clotaire avait la tête couverte par les feuilles vertes du bouquet et c'est vrai que ça ressemblait drôlement à un pot-au-feu. (p. 68)

$$
\begin{aligned}
& \text { "... كلوتر كفت: (آلسست را نغاه كن، حالا /و با آن كالها ريخت خناه دارى بيدا كرده!) آلسست كه /ين حرف } \\
& \text { را شنيد با دسته كل محكم زد روى سر كلوتر. من داد زدم: " كلهام! كلهام را مى شكنيد!) آلسست بشت سر هم با }
\end{aligned}
$$

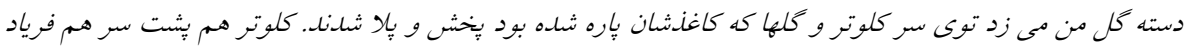

$$
\begin{aligned}
& \text { مى زد: (ادردم نمى آيلا دردم نمى آيدا!).وقتى آلسست دست /ز زدن كلوتر برداشت، سر كلوتر بر شلده بود /ز بركهاى }
\end{aligned}
$$$$
\text { سبز توى دسته كل، سر كلوتر با آن بركها شله بود عين قرمه سبزى.|'(8) (T1, p. 62) }
$$

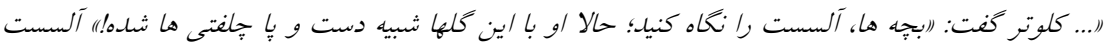$$
\text { هم با دسته كل محكم كوبيد توى سرش. من داد زدم: "ركاهيم! كلهايم را شكستيد!) راست مى كفتم جون آلسست با }
$$$$
\text { دسته كال من يكى عالمه زد تو سر كلوتر و كلها به /ين طرف و آن طرف يرت شد؛ تازه كاغذش هم ياره شد. كلوتر }
$$$$
\text { داد مى زد: "دردم نيامدا دردم نيامد!) وقتى آلسست كتك كاريش تمام شل، سر كلوتر بر از بركهاى سبز شاه بود و }
$$

$$
\text { (T2, p. 74) (1/ستى راستى شبيه ديزى شلده بود.)' }
$$

(8) "Clotaire a dit : "Regarde Alceste, maintenant c'est lui qui a l'air rigolo avec les fleurs." Alors Alceste lui a donné un grand coup sur la tête avec le bouquet. "Mes fleurs ! J'ai crié, vous allez casser mes fleurs !" Alceste donnait sans cesse des coups avec mon bouquet sur la tête de Clotaire, et les fleurs s'éparpillaient, parce que le papier s'était déchiré. Clotaire criait sans arrêt "ça ne me fait pas mal !". Quand Alceste s'est arrêté de donner des coups à Clotaire, il avait la tête couverte par les feuilles vertes du bouquet : la tête de Clotaire avec ces fleurs ressemblaient à du ghormeh sabzi. " (le ghormeh sabzi est un plat composé d'un mélange de légumes et de haricots rouges, de viande d'agneau ou de veau et de citrons secs qui se mange avec du riz)

(9) Clotaire a dit : "Regardez Alceste, maintenant c'est lui qui a l'air d'un maladroit avec des fleurs." Alors Alceste a donné un grand coup sur la tête. J'ai crié "Mes fleurs! Vous allez casser mes fleurs !". J'avais raison parce qu'Alceste donnait des tas de coups sur la tête de Clotaire avec mes fleurs et les fleurs s'envolaient dans tous les sens, et le papier s'était déchiré. Clotaire a crié "ça ne m'a pas fait mal, ça ne m'a pas fait mal." Quand les coups d'Alceste se sont arrêtés, la tête de Clotaire était couverte par les feuilles vertes et ça ressemblait vraiment à du dizi. " (le dizi est un plat iranien qui ressemble un peu à du couscous). 
Le Petit Robert propose la définition suivante pour le pot-au-feu :

"Mets composé de viande et de légumes que l'on fait bouillir dans le "pot" "

Donc ça ressemble plus à ديزى qu'à قرمه سبرى. Quand on lit cette phrase :

$$
\text { " سر كلوتر تر /ز بركهاى سبز شاه بود و راستى راستى شبيه ديزى شله بود.||(10) }
$$

On ne saisit pas le côté humoristique du texte puisqu'on ne trouve pas la ressemblance entre ديزى et la tête d'Alceste couverte de feuilles vertes. En général, on ne met pas de légumes ديزى ديزى دئ (11). On n'arrive pas à imaginer comment la tête de Clotaire ressemble à qui n'est pas vert et ne contient pas de légumes. En conséquence, ce n'est pas drôle pour nous et le texte traduit ne transmet pas le même effet d'humour aux lecteurs de la langue cible. La première traductrice a trouvé un repas plus ressemblant pour les lecteurs cibles. Un

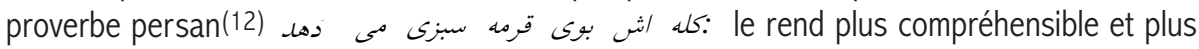
familier pour nous. Ce proverbe qui vient à l'esprit augmente le côté comique du texte, mais c'est encore une sorte de reterritorialisation! Comme les noms utilisés dans le texte (Nicolas, Alceste, Agnan,...) indiquent l'environnement occidental de l'histoire, il est difficile d'imaginer Alceste en train de manger قيزمى سبزى (13) ou (14). Dans ce type de situation, le traducteur doit trouver quelque chose d'universel et de familier pour tous les pays. Par exemple ici, le traducteur pourrait choisir (15) بيتزاى سبزيجات. Ce mot est bien connu de tous et ne constitue pas une reterritorialisation qui déforme l'histoire. بيتزاي سبنزيجات garde également l'environnement occidental du texte. Dans ce cas بيتينزاى سبزيجات est une équivalence dynamique selon Nida.

L'identification du message humoristique est nécessaire pour la traduction car on peut transférer le même message non pas par une traduction mot à mot, mais par une sorte de recréation dans la langue cible.

(10) "Quand les coups d'Alceste se sont arrêtés, la tête de Clotaire était couverte par les feuilles vertes et ça ressemblait vraiment à du dizi. "

(11) Dizi.

(12) "Sa tête sentait le ghormeh sabzi. ", proverbe persan qui désigne quelqu'un qui n'agit pas de manière sage et n'a pas d'idées logiques, qui prend de gros risques.

(13) Dizi.

(14) Ghormeh sabzi.

(15) Pizza de légume. 


\section{L'humour langagier}

Le double sens est l'une des composantes majeures de l'humour. II constitue l'ambiguïté, le jeu de mots, l'ironie et le sarcasme. L'humour basé sur la langue est très difficile, voire impossible à traduire car il s'appuie sur la structure de la langue de départ. Aussi, pour produire le même effet sur le lecteur du texte traduit et transférer le message, on est obligé de reconstruire la même structure dans la langue cible. II est presque impossible de trouver deux langues similaires. De nombreuses particularités linguistiques sont intraduisibles. Ainsi "le Bouillon ", ce sobriquet drôle que Nicolas et ses camarades ont donné à leur surveillant, est un exemple d'humour langagier :

Le Bouillon, c'est un autre surveillant, qu'on appelle comme ça parce qu'il dit toujours : "Regardez-moi dans les yeux", et comme dans le bouillon il y a des yeux, on l'appelle le Bouillon. Ce sont les grands qui ont trouvé ça. (Sempé-Goscinny, 1960, p. 148)

$$
\begin{aligned}
& \text { "ا آقاى دوبن يك ناظم ديخر /ست و ما /سمش را بويون(آبكوشت) كذاشته /يم، براى /ينكه هميشه مى كويل: } \\
& \text { "انكاه كنيد، توى جشمهاى من" و خون توى بويون هم، وقتى كه مى جوشل، جشم درست مى شود، بهش بويون مى }
\end{aligned}
$$

$$
\text { كوييم. /ين را بزركها كشف كرده /ند.،(16) (T1, p. } 139)
$$
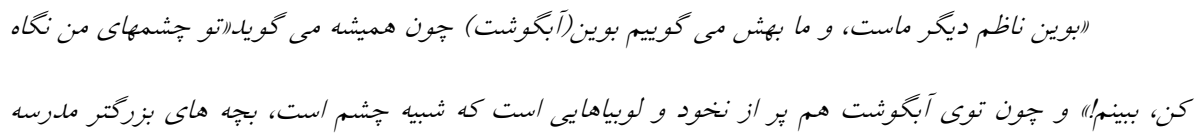

$$
\text { برايش /ين اسم را بيدا كرده /ند.ب(17) (T2, p. } 158
$$

(16) " Le bouillon est un autre surveillant qu'on appelle le bouillon, parce qu'il dit toujours : "regardez dans mes yeux" et comme dans le bouillon, quand il bout on voit des yeux donc on l'appelle le Bouillon. Ce sont les grands qui ont découvert ça."

(17) "Le bouillon est notre autre surveillant et on l'appelle bouillon (abgouchte) parce qu'il dit toujours "Regarde dans mes yeux" et comme abgouchte est plein de haricots et de pois chiches qui ressemblent aux yeux, les grands de l'école ont trouvé ce surnom pour lui. " 
Chaque traductrice a interprété à sa manière le mot "Bouillon ". Comme on le voit, elles ont été obligées d'ajouter certaines explications pour clarifier le sens, comme le mot entre paren-

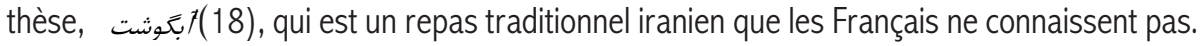
II semble que les traductrices voulaient reterritorialiser ce mot pour le rendre plus accessible aux enfants de la langue cible. Mais le problème est que le mot "Bouillon " est ici un nom propre qui a un sens humoristique particulier. Comment peut-on garder à la fois le nom propre tel qu'il existe dans le texte original et le côté humoristique qui réside dans le sens de ce nom caché aux yeux du lecteur cible ? Le mot " bouillon " en français n'a aucun besoin d'explication dans la version originale. En lisant le texte, le lecteur de la langue source saisit facilement le sens, "le bouillon " étant un plat traditionnel très connu dans lequel la graisse forme "des yeux". En persan, les traducteurs ont dû transposer et utiliser une référence aux pois chiches comparés à des yeux pour rendre l'allusion compréhensible. En effet, en lisant le mot بَآبكوشت le lecteur de la langue d'arrivée ne comprend pas et il lui faut une explication. Le lecteur de la langue cible saisit, grâce aux explications ajoutées, l'humour caché derrière ce mot. Amir Hossein Mehdi Zâdeh, un autre traducteur des récits du Petit Nicolas a choisi كله جوش (Mehdi Zâdeh, 2005, 3) comme équivalent, qui convient bien pour ce terme.

\section{Humour et traduction au contact des cultures}

Selon C. Lévi-Strauss :

Toute culture peut être considérée comme un ensemble de systèmes symboliques au premier rang desquels se placent le langage, les règles matrimoniales, les rapports économiques, l'art, la science, la religion. (Hess, Wulf, 1999, p. 47).

La langue s'inscrit dans un contexte culturel. II existe un lien entre une langue et une façon de penser, qui elle-même influence la culture. Les langues que nous parlons donnent des informations sur nos identités, nos idéologies et nos histoires. "Une définition du langage est toujours, implicitement ou explicitement, une définition des êtres humains dans le monde. " (Williams, 1977, p. 21)

Nida a lui aussi souligné l'importance des éléments culturels dans la traduction :

Linguistic features are not the only factors which must be considered. In fact, the cultural elements may be even more important. (Nida, 1969, p. 130)

Comme nous l'avons indiqué plus haut, selon Bergson, le comique est le propre de l'homme. Les peuples de tous les pays et de toutes les cultures rient. Les sujets comiques diffèrent selon

(18) Abgouchte, plat iranien, autre nom du dizi.

(19) Kalehdjoche, un plat iranien. 
les conditions sociales, l'âge, la géographie et la culture des lecteurs ou des auditeurs. lls diffèrent aussi parfois au sein d'un même pays. Par exemple, ce qui est drôle pour les peuples du nord de l'Iran ne l'est pas forcément pour ceux qui viennent du sud. Mais le point commun entre toutes les manifestations humoristiques réside dans le fait que ce qu'on lit, entend ou voit est inattendu. Voici un exemple tiré de l'un des récits du Petit Nicolas, quand Geoffroy demande au photographe :

"C'est quoi, votre appareil ?"

Le photographe lui dit :

"C'est une boîte d'où va sortir un petit oiseau, bonhomme. "(Sempé-Goscinny, 1960, p. 10)

Geoffroy commence à expliquer le fonctionnement de son appareil :

"Il est vieux votre engin, a dit Geoffroy, mon papa il m'en a donné un avec parasoleil, objectif à courte focale, téléobjectif, et, bien sûr, des écrans... " (Ibid., p. 10)

Geoffroy surprend le lecteur par les informations complètes qu'il donne.

Les textes humoristiques qui nous reconduisent dans nos milieux et qui nous dévoilent les laideurs de la société dans laquelle nous vivons sont uniquement imaginables et compréhensibles par nous. Nous n'avons pas besoin d'explications complémentaires pour les comprendre. Tandis que pour les personnes extérieures à notre société, il faut ajouter des explications et des interprétations. Ces personnes risquent alors de passer à côté de l'humour du texte, car la brièveté est une caractéristique importante des textes humoristiques. Par exemple, si nous racontons une blague avec beaucoup d'explications, l'auditeur ne rira pas. C'est pour cette raison que certaines blagues dans une langue donnée ne sont pas drôles pour ceux qui ne vivent pas dans le pays.

[...] toute culture est ethnocentrique dans la mesure où ceux qui la partagent ressentent les règles de leur propre culture comme "naturelles " ou "normales", et les modes de pensée et les comportements des autres cultures comme "étranges ", " non naturels ", voire " déviants ". " (Critchley, 2005, p. 107). La traduction est l'un des moyens de transformation des cultures. Celle-ci transfère aussi la pensée des nations.

L'humour est souvent considéré comme intraduisible, et pourtant on le traduit. Le texte traduit appartient aux lecteurs de la langue cible. II faut donc parfois le changer, l'adapter en fonction des exigences de ces lecteurs. Les notes de bas de pages peuvent ajouter des informations complémentaires pour aider le lecteur à comprendre les différences culturelles. Mais le fait de lire ces notes peut interrompre la lecture du texte. C'est au traducteur de distinguer où il faut ajouter les notes pour ne pas stopper la lecture. Parfois, il est préférable d'ajouter les explications nécessaires dans le corps du texte. Mais cette stratégie est-elle applicable aux textes humoristiques ? Ce qui est important dans ce type de textes, c'est la brièveté. Le degré des changements opérés au moment de la traduction dépend de la différence entre les deux cultures. 
Parfois, au lieu de donner des explications, il est possible d'ajouter un mot modificateur pour éviter les explications détaillées. Voici un exemple du Petit Nicolas :

Rufus a dit qu'il ne connaissait pas la fable par cœur, mais qu'il savait à peu près de quoi il s'agissait et il a commencé à expliquer que c'était l'histoire d'un corbeau qui tenait dans son bec un roquefort. (Sempé-Goscinny, 1960, p. 45)

"روفوس كه دوست من است و باباش پاسبان است، كفت نمى تواند قصه را از بر بخواند، ولى تقريبا مى داند كه

داستان كلاغى است كه يك تكه بنير روكفور را به منقارش گرفته بود (.)(T1, p. 41)(

"روفو كفت كه حكايت را از حفظ بلد نيست اما تقريبا مى داند كه موضوع آن جيست و شروع كرد به تعريف كردن

كه داستان يك كلاغ است كه يك قالب بنير روكفور به نوكش كرفته بود.1) (21) (T2, p. 53)

Dans cet exemple, nous constatons que les deux traductrices ont ajouté le terme "fromage " dans leurs traductions. II s'agit d'un terme modificateur. Parce qu'en France, quand on parle de "roquefort ", il est clair qu'il s'agit d'un fromage. Mais le lecteur persan ne comprend pas le sens de "roquefort ". II faut donc ajouter pour lui un terme modificateur.

Le traducteur doit donc avoir une connaissance suffisante des deux cultures. Le fait de garder la couleur locale du texte original et sa culture est l'un des axes de fidélité. Ainsi, dans le cas de la traduction de textes littéraires et particulièrement de textes humoristiques, il s'agit d'établir un pont entre des cultures très différentes l'une de l'autre. Les difficultés liées à la traduction sont d'une part d'ordre linguistique et stylistique, et d'autre part d'ordre sociolinguistique.

Parfois on ne trouve pas l'équivalent d'un mot dans la culture cible, parce que certaines réalités extralinguistiques n'existent pas dans la langue du traducteur. Cela produit un décalage culturel entre le texte source et le texte d'arrivée. Dans ce cas, un bon traducteur est celui qui peut adapter l'œuvre traduite aux exigences de la culture du pays d'accueil en préservant l'essence du texte. Mais pour comprendre un texte traduit, il est nécessaire d'avoir un minimum de connaissance des réalités extralinguistiques auxquelles renvoie le texte source.

Souvent l'humour, le trait caractéristique des ouvrages humoristiques, ne passe pas par le filtre de la traduction. Selon Nida, si un traducteur n'arrive pas à trouver un équivalent pour

(20) "Rufus, un ami à moi qui a un père agent de police, a dit qu'il ne pouvait pas dire le conte par cœur, mais il savait à peu près qu'il s'agissait de l'histoire d'un corbeau qui tenait dans son bec une part de fromage de roquefort. "

(21) " Rufus a dit qu'il ne connaissait pas le conte par cœur mais il savait à peu près le sujet et il a commencé à raconter qu'il s'agissait de l'histoire d'un corbeau qui tenait une boule de fromage de roquefort dans son bec." 
un mot dans la langue cible, ou si le mot n'existe pas dans cette langue, il peut " trouver un mot qui a la même valeur et qui est le plus proche équivalent dans les langue et culture cibles " (Solhdjo, 1998, p.79). Parfois le traducteur est obligé d'ajouter ou de supprimer quelques éléments du texte original pour éclaircir les éléments culturels de la langue source. II faut savoir qu'il y a toujours une perte ou une déformation dans toute situation de communication, en particulier quand il s'agit de traduire dans une langue cible des termes régionaux empreints de couleur locale.

\section{Conclusion}

La traduction et la culture ont une relation très étroite. Pour comprendre le langage humoristique de chaque nation, il faut connaître sa culture. Dans la traduction des textes humoristiques, il faut recréer l'idée humoristique de l'auteur et l'effet du texte original de sorte que le lecteur cible saisisse l'humour. La traduction de l'humour va au-delà de la traduction des mots. L'une des difficultés de la traduction de textes humoristiques pour enfants est la nécessité de transformer un langage à la fois enfantin et humoristique. Le traducteur doit avoir un sens inné de l'humour, une certaine créativité et une bonne dose d'imagination pour transférer correctement l'effet humoristique de ce type de textes. II doit choisir des mots, des phrases et des structures grammaticales qui puissent produire les mêmes images humoristiques que le texte original, de sorte qu'elles soient palpables et plaisantes pour les lecteurs cibles. On a vu que les procédés qui produisent l'effet humoristique des récits du Petit Nicolas reposent souvent sur les réalités extralinguistiques. Nous avons constaté quelques problèmes linguistiques dans la traduction de cette œuvre mais cela n'est pas une raison suffisante pour la rendre intraduisible. Des particularités telles que les répétitions d'un mot dans des situations différentes et les humours culturels et langagiers sont parfois à l'origine des difficultés rencontrées lors de la traduction du Petit Nicolas en persan. On a considéré que le succès en revenait au langage imagé de Goscinny. Donc, les textes humoristiques pour enfants sont traduisibles si le sujet, la langue, la structure des phrases, le style, etc. sont au service de la production d'images humoristiques. Nous constatons l'omniprésence de l'image dans le monde d'aujourd'hui à travers Internet, les jeux vidéo et les différents produits audio-visuels. Même les livres électroniques vont remplacer les livres papier. Par ce travail de recherche, nous avons voulu utiliser la théorie de Nida pour présenter un début de théorisation de la traduction de textes humoristiques pour enfants : une bonne traduction d'un texte humoristique pour enfants est celle qui produit la même image humoristique pour le lecteur de la langue d'arrivée que le texte original pour son lecteur. 
Sevil ZEYNALIGARGARI est actuellement étudiante en M2 de LTMT (Lexicologie, terminologie multilingue et traduction) à l'université Lumière Lyon II. Elle est titulaire d'un Karshenassi arshad (diplôme de niveau bac +6$)$ de traduction française à l'Université de Téhéran et traductrice d'un roman intitulé Baisers de cinéma d'Eric Fottorino en persan (publication prévue en juillet 2011). Elle collabore avec des maisons de traduction et a traduit différents textes dans les domaines de la médecine, de l'ingénierie, etc. en Iran.

Farideh ALAVI est maître-assistant à la Faculté des langues et des littératures étrangères, université de Téhéran, Iran. Pour plus d'informations, consulter le site :

http://ffll.ut.ac.ir/Group_detTeacher_User.asp?codePerson=17\&codeGroup $=5$

\section{Bibliographie}

BERGSON Henri, 1959, Le rire, essai sur la signification du comique, Paris, PUF.

DOUGLAS Virginie, 2007, "Traduire l'intertextualité en littérature pour la jeunesse ", Palimpsestes, $\mathrm{n}^{\circ} 18$.

EBRAHIMI Nâder, 1975, Écrire en persan pour enfants, Téhéran, Âgâh.

ESCARPIT Robert, 1994, L'Humour, Paris, PUF,

FROMILHAGUE Catherine, SANCIER Anne, 1991, Introduction à l'analyse stylistique, Paris, Bordas. GOSCINNY René, 1960, Le Petit Nicolas, Paris, Denoël.

HESS Remi, WULF Christophe, 1999, Parcours, passages et paradoxes de l'interculturel, Paris, Anthropos.

HORRI Abolfazl, 2007, "Introduction au principe et à la théorie de la traduction de l'humour ", Étude de la traduction, $\mathrm{n}^{\circ} 14$.

JUN Xu, 1999, "Réflexions sur les études des problèmes fondamentaux de la traduction ", Meta : Journal des traducteurs, $\mathrm{n}^{\circ} 48$.

KRITCHLEY Simon, 2006, On Humour, traduit par Soheyl Sommi, Téhéran, Ghoghnoos.

MOHÉBI Maryame, "Les types d'humour dans la littérature pour enfants ", Le livre du mois, $n^{\circ} 552$.

MOUNIN Georges, 1963, Les problèmes théoriques de la traduction, Paris, Gallimard.

NIDA Eugene A. et TABER Charles R., 1969, The Theory and Practice of Translation, Leiden, E.-J. Brill. 
NIDA E. A., Contexts in Translating, 2001, Amsterdam \& Philadelphia, John Benjamins.

HESS Rémi, WULF Christophe, Parcours, passages et paradoxes de l'interculturel, Paris, Anthropos, 1999

SEMPÉ-GOSCINNY, 2002, Le Petits Nicolas, traduit par Âfagh Hâmed Hâchémi, Téhéran, Sorouche.

SEMPÉ-GOSCINNY, 2002, Le Petits Nicolas, traduit par Vida Saâdat, Téhéran, Hermesse.

SEMPÉ-GOSCINNY, 2006, Le Petits Nicolas et les copains, traduit par Amir Hossein MehdiZadeh, Téhéran, Hermesse.

SOLHDJO Ali, 1999, Forum et traduction, Téhéran, Markaz.

WILLIAMS Raymond, 1977, Marxism and Literature. Oxford, Oxford University Press.

XIAOYI Yuan, 1999, "Débat du siècle : fidélité ou recréation ", Meta : journal des traducteurs, $n^{\circ} 44$.

http://www.crdp.ac-creteil.fr/telemaque/comite/JPGourevitch.htm, consulté le 28 février 2009. 